\title{
Photodynamic Therapy Rescue for Subretinal Fluid Exacerbation After Focal Laser Treatment in Idiopathic Central Serous Chorioretinopathy
}

\author{
Theodore Leng*, Steven R. Sanislo and Robert L. Jack \\ Department of Ophthalmology, Stanford University School of Medicine, Stanford, California, USA
}

\begin{abstract}
Purpose: To report a case of subretinal leakage after focal laser treatment for idiopathic central serous chorioretinopathy (ICSC). This rare complication was successfully treated with photodynamic therapy (PDT).

Methods: Interventional case report.

Results: A 36-year-old male presented with ICSC in his right eye. After a period of observation without resolution, he was treated with focal laser. That treatment resulted in a massive exacerbation of his subretinal fluid. PDT was successfully used to treat the severe exacerbation with rapid resolution of the subretinal fluid, improvement in visual acuity, decreased leakage on fluorescein angiography, and reduction of subretinal fluid on ophthalmoscopic exam and by optical coherence tomography.

Conclusions: Ophthalmologists should consider the use of PDT in cases where focal laser causes an exacerbation of subretinal fluid in ICSC.
\end{abstract}

Keywords: Idiopathic central serous chorioretinopathy, focal laser, photodynamic therapy.

\section{INTRODUCTION}

Idiopathic central serous chorioretinopathy (ICSC) is a relatively common disorder of young or middle age individuals with abnormalities of the retinal pigment epithelium (RPE), resulting in focal areas of serous retinal detachment and pigment epithelial detachment [1]. Fluorescein angiography often demonstrates focal areas of leakage at the level of the RPE with pooling of dye into the subretinal or sub-pigment epithelial space. In most cases the leakage resolves spontaneously with gradual return of good visual function. If there is chronic or recurrent leakage treatment may be indicated. In such cases, light focal laser photocoagulation to the area of RPE abnormality and leakage may be employed [2-5].

Here, we report a case of ICSC where focal laser photocoagulation resulted in a dramatic increase in fluid leakage with severe worsening of the serous macular detachment. Subsequent treatment with photodynamic therapy (PDT) resulted in prompt resolution of the leakage and reduction of subretinal fluid.

\section{CASE REPORT}

A 36 year-old Caucasian male presented with two days of blurring of the central vision in the right eye with a bestcorrected visual acuity of 20/20-2. Four years ago, he had ICSC in the left eye that was successfully treated with reduced fluence PDT after an ineffective focal laser session.

Dilated fundus examination of the right eye disclosed an area of serous detachment in the superonasal macula

*Address correspondence to this author at the Eye Institute at Stanford, Stanford University School of Medicine, Palo Alto, CA 94303, USA; Tel: (650) 498-4264; Fax: (650) 565-8297; E-mail: tedleng@stanford.edu extending toward the center of the fovea (Fig. 1A). Optical coherence tomography (OCT) confirmed the presence of subretinal fluid in the superonasal macula just sparing the foveal center (Fig. 1B). Fluorescein angiography showed a focal spot of leakage at the level of the RPE in the superonasal macula with pooling of dye into the area of subretinal fluid seen on ophthalmoscopy (Figs. 1C, D). The fellow eye had a best-corrected visual acuity of $20 / 20$ and dilated fundus examination revealed RPE hyperpigmentation in the inferior foveal region without subretinal fluid.

A diagnosis of recurrent ICSC was made and options for treatment were discussed including: observation, focal laser photocoagulation, or PDT. After an initial observation period of three months without symptomatic or funduscopic change, a decision was made to treat with focal laser photocoagulation. Twelve $200 \mu \mathrm{m}$ light-intensity laser spots were applied with an argon laser to the area of leakage utilizing $200-400 \mathrm{~mW}$ at $100 \mathrm{msec}$ duration. Ten days later the patient returned having noted a dramatic worsening of vision. At this point, the visual acuity in the right eye measured 20/400 without correction and improved to 20/150 with a spherical correction of +0.75 diopters.

Repeat funduscopic examination disclosed a dramatic increase in subretinal fluid (Fig. 2A) that was confirmed by OCT (Fig. 2B). Fluorescein angiography showed a new area of leakage at the inferior edge of the laser scar (Figs. 2C, D). Because of the increased leakage a decision was made to treat with PDT. Verteporfin $6 \mathrm{mg} / \mathrm{m}^{2}$ was administered intravenously and a $600 \mu \mathrm{m}$ laser spot was applied to the area of leakage for 83 seconds at half-fluence. One week later visual acuity had improved to 20/60 without correction and the subretinal fluid had decreased in size both on exam and by OCT (Figs. 3A, B). Furthermore, there was no longer 

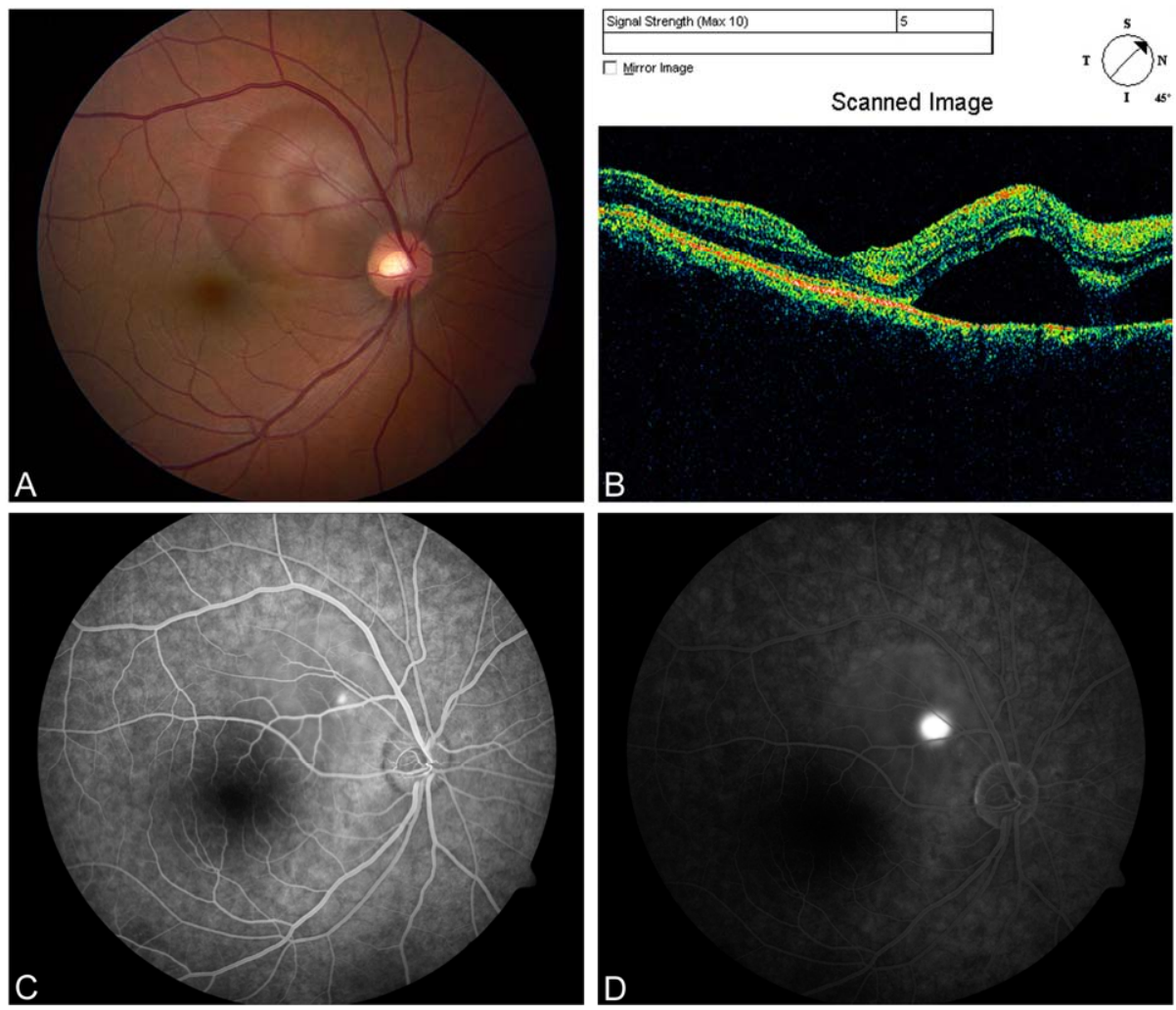

Fig. (1). (A) Color fundus photograph of the right eye showing a well demarcated area of subretinal fluid in the superonasal macula. (B) Optical coherence tomography of the right eye showing subretinal fluid within the superior macula extending very close to the foveal center. (C) Early frame fluorescein angiogram of the right eye showing focal area of leakage in the superonasal macula. (D) Late frame fluorescein angiogram of the right eye showing increasing leakage with pooling into the area of subretinal fluid.
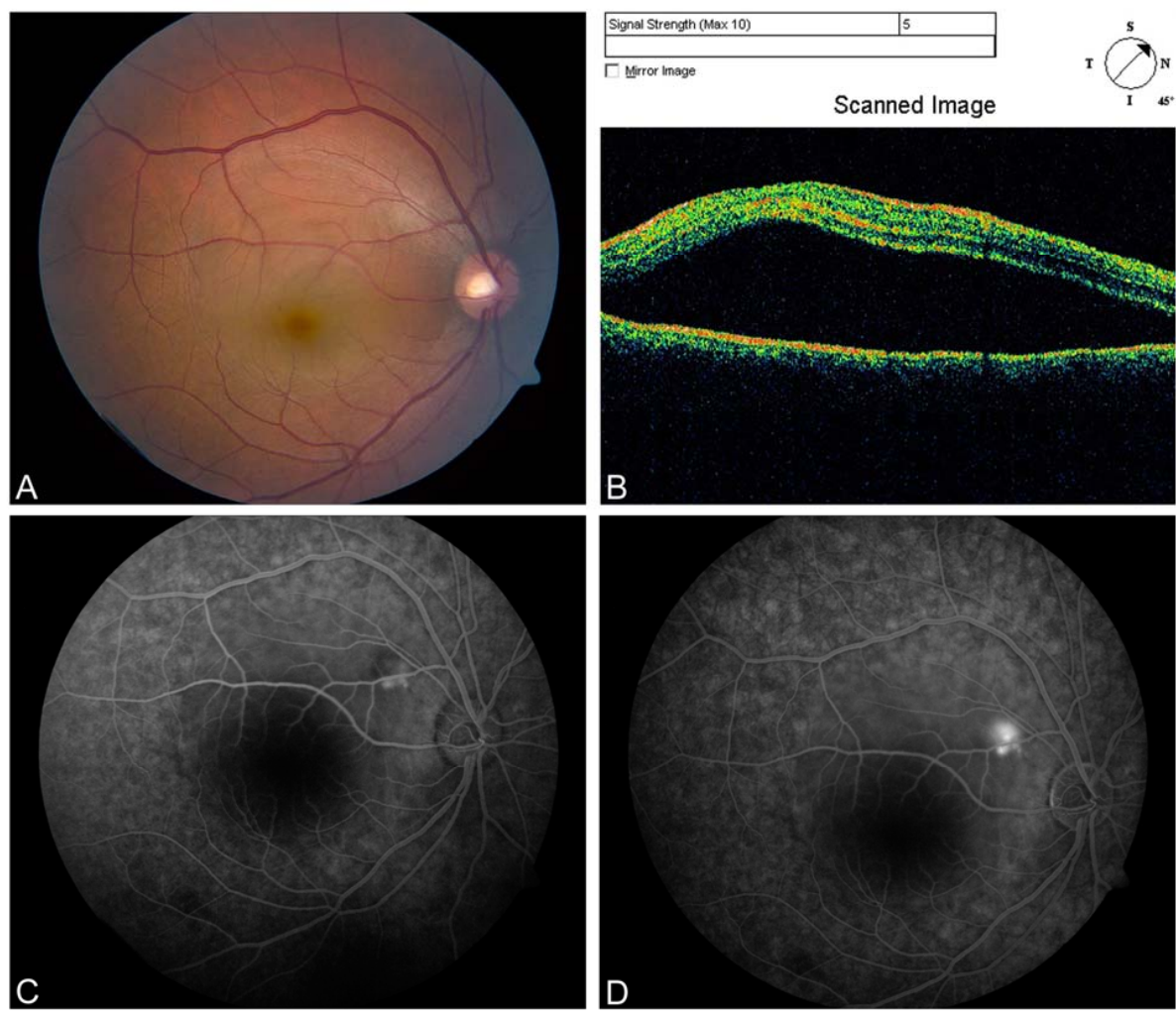

Fig. (2). (A) Color fundus photograph and (B) optical coherence tomography of the right eye showing a dramatic increase in subretinal fluid after focal laser photocoagulation. (C) Early and (D) late frame fluorescein angiograms of the right eye showing a new area of leakage just below the area treated with laser photocoagulation with later leakage and pooling of dye. 

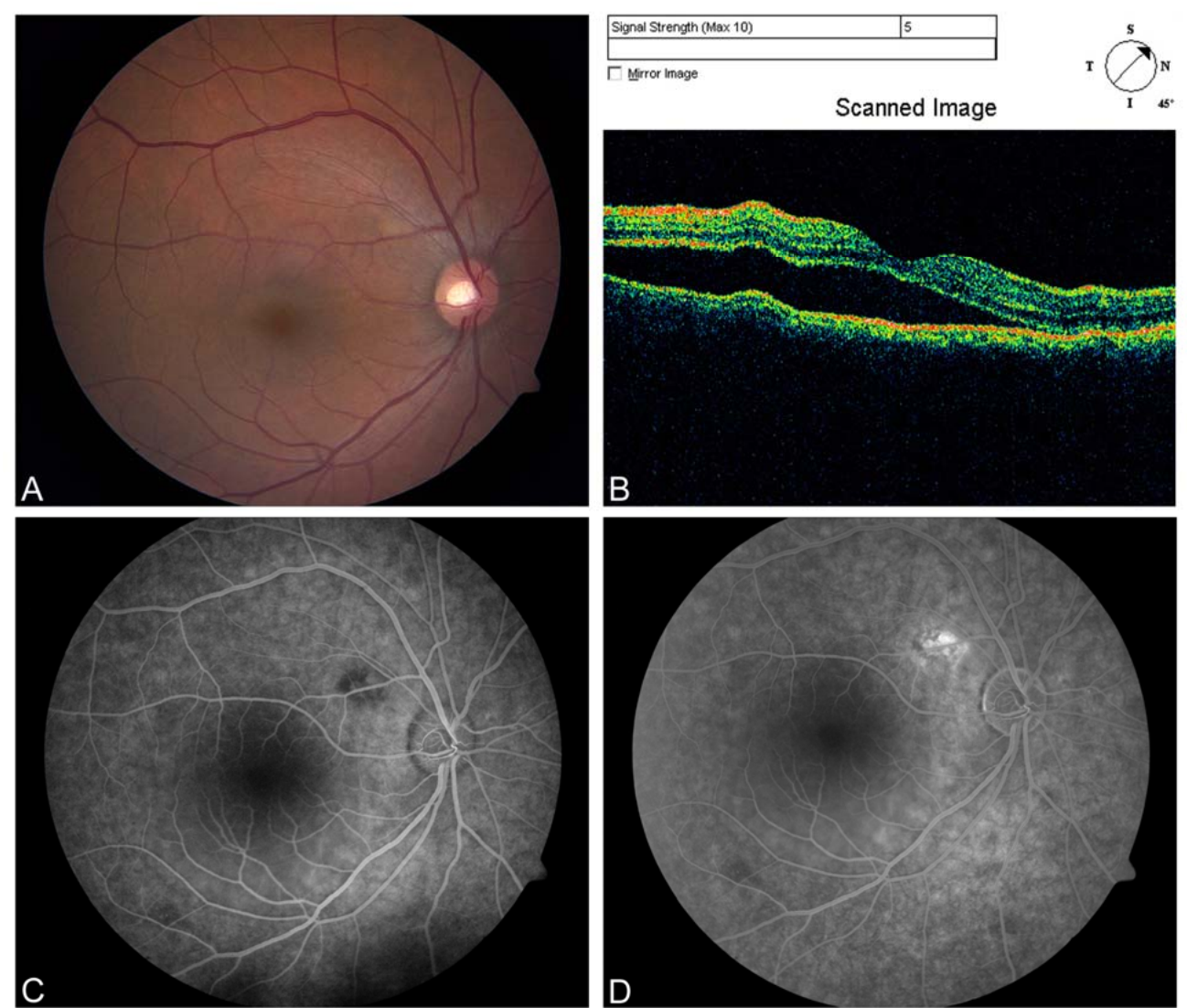

Fig. (3). (A) Color fundus photograph and (B) optical coherence tomography of the right eye showing a reduction in the amount of subretinal fluid after PDT. (C) Early and (D) late frame fluorescein angiograms showing mild dye transmission in area treated by focal laser with late staining of the laser scar in the superonasal macula.

leakage on fluorescein angiography (Figs. 3C, D). At 3 months after PDT visual acuity recovered to 20/20. Funduscopic exam and OCT showed no subretinal fluid and FA continued to show no leakage (Fig. 4). The patient continues to do well without further recurrence of leakage at after 12 months of follow up.

\section{DISCUSSION}

Most cases of ICSC do not require any intervention and patients regain good visual function [1]. Treatment is indicated in patients with recurrent leakage because multiple episodes of leakage can result in permanently reduced vision. The most common intervention is focal laser photocoagulation, which has been shown to enhance the resolution of leakage and absorption of subretinal fluid; however, the mechanism of action is poorly understood.

PDT with verteporfin has also been utilized for treatment of some cases of ICSC [6-10]. Due to the fact that there is relatively little data on the safety and efficacy of PDT in patients with ICSC, this treatment is usually reserved for cases that have not resolved spontaneously and either are not candidates for focal laser (due to poorly defined leakage or leakage too close to the center of the fovea) or have failed treatment with focal laser. The mechanism by which PDT reduces leakage in ICSC is also poorly understood; however, it may help to decrease the choroidal hyperpermeability that is the proposed pathogenetic mechanism of this disease and can be observed during indocyanine green angiography [11].
This case is unique in that focal laser photocoagulation resulted in no improvement in leakage four years prior in the patient's left eye, and a dramatic worsening of leakage in the fellow eye described in this report. Focal laser treatment for ICSC has been reported to have some risk, including inadvertent treatment in the fovea and development of secondary choroidal neovascularization (CNV) at the laser scar $[12,13]$. To the best of our knowledge the complication described here (dramatically increased subretinal fluid) has not been previously reported. It is highly unlikely that the increased fluid in our patient represented development of CNV because it occurred immediately after the laser treatment; however, it is unclear why laser treatment resulted in exacerbation of leakage. Fortunately, in both eyes of this patient, treatment with PDT resulted in rapid and complete resolution of macular leakage and serous detachment, although PDT has also been associated with the possible side effect of choroidal ischemia.

Most patients with ICSC never require intervention. In those that do require treatment focal laser photocoagulation will likely remain the most common treatment. However, PDT has been found to be effective in this disorder, and use of this treatment will likely increase. Specifically, one should consider the use of PDT in cases where there is increased leakage of subretinal fluid after focal laser treatment. Additionally, for patients with ICSC where focal laser was found to be unsuccessful in one eye, consideration should be given to using PDT as initial therapy if the fellow eye should it require treatment. 

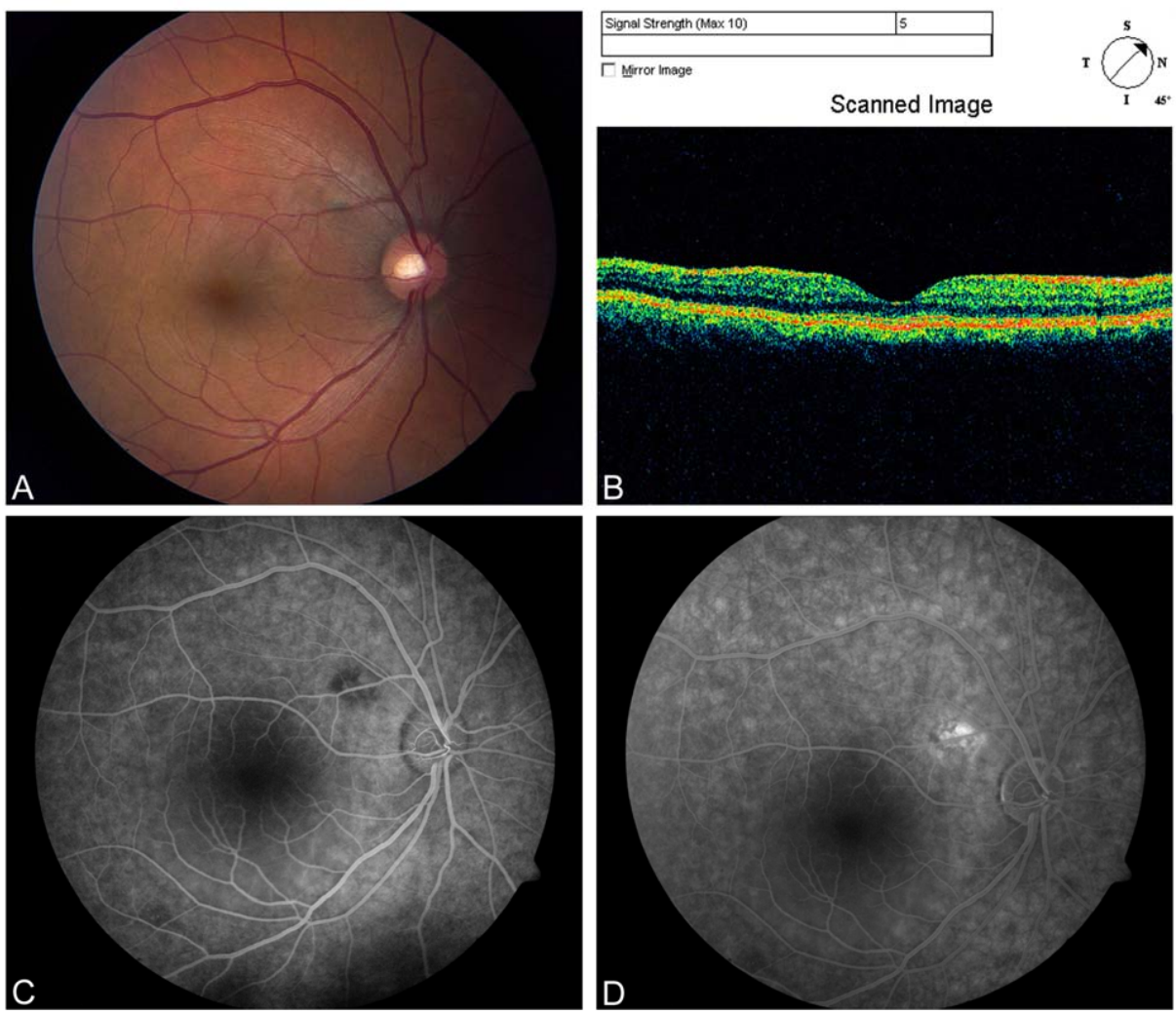

Fig. (4). (A) Color fundus photograph and (B) optical coherence tomography of the right eye showing complete resolution of subretinal fluid and a faint pigmented scar in the location of prior laser photocoagulation. (C) Early and (D) late frame fluorescein angiograms showing early hypofluorescence in the area of prior leakage and late staining of the laser scar with no leakage.

\section{DISCLOSURES AND CONFLICTS OF INTEREST}

None of the authors has any commercial or financial conflicts with any of the materials or companies mentioned in this manuscript.

The material in this manuscript has not previously been presented elsewhere.

\section{REFERENCES}

[1] Bouzas EA, Karadimas P, Pournaras CJ. Central serous chorioretinopathy and glucocorticoids. Surv Ophthalmol 2002; 47(5): 431-48.

[2] Ficker L, Vafidis G, While A, Leaver P. Long-term follow-up of a prospective trial of argon laser photocoagulation in the treatment of central serous retinopathy. Br J Ophthalmol 1988; 72(11): 829-34.

[3] Gass JD. Photocoagulation treatment of idiopathic central serous choroidopathy. Trans Sect Ophthalmol Am Acad Ophthalmol Otolaryngol 1977 83(3 Pt 1): 456-67.

[4] Landers MB, 3rd, Shaw HE, Jr, Anderson WB, Jr, Sinyai AJ. Argon laser treatment of central serous chorioretinopathy. Ann Ophthalmol 1977; 9(12): 1567-72.

[5] Robertson DM, Ilstrup D. Direct, indirect, and sham laser photocoagulation in the management of central serous chorioretinopathy. Am J Ophthalmol 1983; 95(4): 457-66.

[6] Battaglia Parodi M, Da Pozzo S, Ravalico G. Photodynamic therapy in chronic central serous chorioretinopathy. Retina 2003; 23(2): 235-7.
[7] Lai TY, Chan WM, Li H, Lai RY, Liu DT, Lam DS. Safety enhanced photodynamic therapy with half dose verteporfin for chronic central serous chorioretinopathy: a short term pilot study. Br J Ophthalmol 2006; 90(7): 869-74.

[8] Valmaggia C, Niederberger H, Photodynamic therapy in the treatment of chronic central serous chorioretinopathy. Klin Monbl Augenheilkd 2006; 223(5): 372-5

[9] Yannuzzi LA, Slakter JS, Gross NE, et al. Indocyanine green angiography-guided photodynamic therapy for treatment of chronic central serous chorioretinopathy: a pilot study. Retina 2003; 23(3): 288-98.

[10] Chan WM, Lai TY, Lai RY, Liu DT, Lam DS. Half-dose verteporfin photodynamic therapy for acute central serous chorioretinopathy: one-year results of a randomized controlled trial. Ophthalmology 2008; 115(10): 1756-65.

[11] Inoue R, Sawa M, Tsujikawa M, Gomi F. Association between the efficacy of photodynamic therapy and indocyanine green angiography findings for central serous chorioretinopathy. Am J Ophthalmol 2010; 149(3): 441-6 e1-2.

[12] Matsunaga $H$, Nangoh $K$, Uyama $M$, Nanbu $H$, Fujiseki $Y$, Takahashi K. Occurrence of choroidal neovascularization following photocoagulation treatment for central serous retinopathy. Nippon Ganka Gakkai Zasshi 1995; 99(4): 460-8.

[13] Simon P, Glacet-Bernard A, Binaghi M, Coscas G, Soubrane G. Choroidal neovascularization as a complication following laser treatment of central serous chorioretinopathy. J Fr Ophtalmol, 2001. 24(1): p. 64-8.

This is an open access article licensed under the terms of the Creative Commons Attribution Non-Commercial License (http: //creativecommons.org/licenses/by$\mathrm{nc} / 3.0 /$ ) which permits unrestricted, non-commercial use, distribution and reproduction in any medium, provided the work is properly cited. 